\title{
ReaR
}

\section{¿Usamos los dispositivos supraglóticos en rescate de VAD como nos recomiendan las guías?}

Artículo original: J. L. D. Thomsen, A. K. Nørskov and C. V. Rosenstock. Supraglottic airway devices in difficult airway management: a retrospective cohort study of 658,104 general anaesthetics registered in the Danish Anaesthesia Database. Anaesthesia 2019, 74, 151-157 doi:10.1111/anae.14443 (

Fernández Vaquero MA, Fernández Elías E, Álvarez Fernández M.

Clínica Universidad de Navarra, Madrid.

\section{Resumen}

Las indicaciones para el uso de dispositivos supraglóticos se han ido ampliando con el tiempo y hoy en día han tomado un papel destacado en el manejo de la vía aérea difícil dentro de los diferentes algoritmos de las sociedades científicas. Este estudio trata de evaluar si realmente se están poniendo en práctica estos algoritmos(y por tanto el uso de estos dispositivos como rescate) en el día a día médico. Para ello se evalúa un estudio de cohorte retrospectivo de la base de datos danesa, sobre actos anestésicos que comprende un periodo extendido de 2008 a 2012 analizando 658104 anestesias generales de pacientes adultos. Se presta especial atención a los pacientes que presentaron vía aérea difícil, y el manejo que se realiza para asegurar la oxigenación y la vía aérea. Tras análisis estadísticos se concluye que, los dispositivos supraglóticos no son ampliamente utilizados como dispositivos de rescate en pacientes con vía aérea difícil, a pesar de su relevante papel en los algoritmos de manejo de esta.

\section{Introducción}

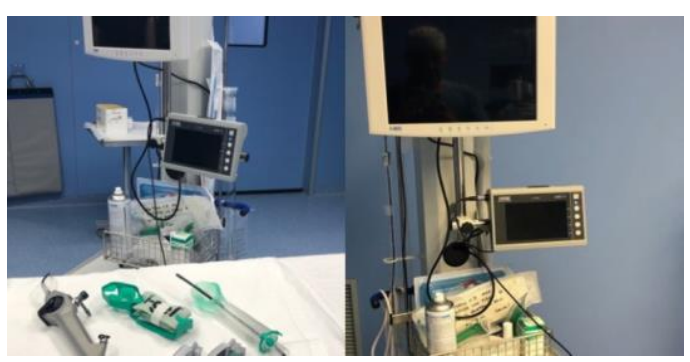

Las indicaciones para el uso de dispositivos supraglóticos se han ido ampliando con el tiempo y hoy en día han tomado un papel destacado en el manejo de la vía aérea difícil dentro de los diferentes algoritmos de las sociedades científicas. Este estudio trata de evaluar si realmente se están poniendo en práctica estos algoritmos(y por tanto el uso de estos dispositivos como rescate) en el día a día médico. Para ello se evalúa un estudio de cohorte retrospectivo de la base de datos danesa, sobre actos anestésicos que comprende un periodo extendido de 2008 a 2012 analizando 658104 anestesias generales de pacientes adultos. Se presta especial atención a los pacientes que presentaron vía aérea difícil, y el manejo que se realiza para asegurar la oxigenación y la vía aérea. Tras análisis estadísticos se concluye que, los dispositivos supraglóticos no son ampliamente utilizados como dispositivos de rescate en pacientes con vía aérea difícil, a pesar de su relevante papel en los algoritmos de manejo de esta.

\section{Resumen}

$\underline{\text { Objetivo }}$

Objetivo primario: utilizar la base de datos danesa para describir el uso de los 
dispositivos supraglóticos en la gestión de los pacientes con vía aérea difícil.

Objetivos secundarios: 1)Tasa de éxito de colocación de Dispositivo Supraglótico (DSG) en procedimientos con vía aérea difícil, 2) proporción de casos "no intubable, no ventilable con mascarilla facial" donde se intentaba la colocación de un DSG, 3) tasa de éxito en estas situaciones y 4) proporción de casos donde había una VAD anticipada y se usaron DSG para su gestión.

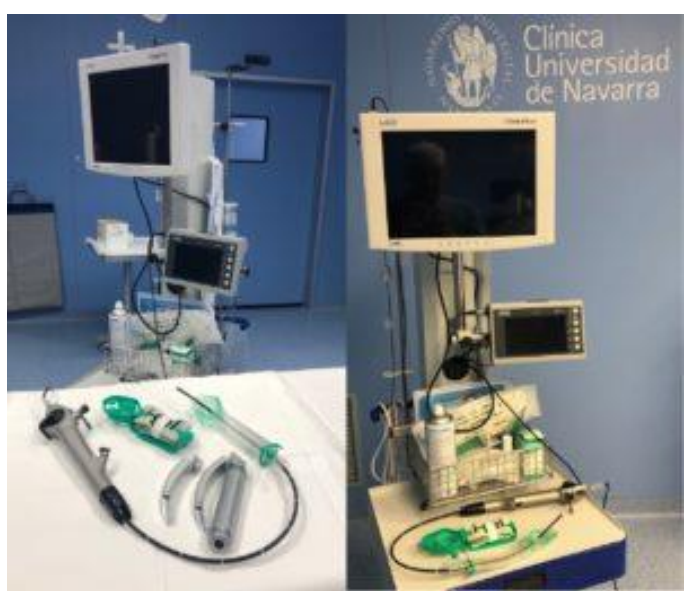

Imagen 1: Carro VAD

\section{Material y métodos}

Utilizando la base danesa de anestesia (datos recopilados entre 2008-2012), en la cual colaboran 43 departamentos de Anestesia de hospitales públicos $\mathrm{y}$ privados, correspondiente a aproximadamente el $75 \%$ de todos los departamentos del país. Los datos registrados en cada paciente fueron: edad, sexo, altura, peso, ASA, prioridad quirúrgica (electiva o urgente), ventilación con mascarilla facial (clasificada en no realizada, fácil grado 1-2 de la escala de HAN o difícil grado 3-4 de HAN) y laringoscopia directa considerándose dificultad de intubación en los pacientes con una puntuación $\geq 2$ y/o dificultad de ventilación con mascarilla facial, e incluyéndose en este estudio a los pacientes que tenían una puntuación $\geq 3 \quad(0$ laringoscopia no realizada, 1 laringoscopia directa en dos ocasiones, 2 laringoscopia directa hasta en tres ocasiones con diferentes dispositivos o palas, 3 más de 3 intentos de laringoscopia directa, 4 intubación traqueal fallida).

\section{$\underline{\text { Resultados }}$}

De un total de 447.037 procedimientos que recibieron 658.104 anestesias a lo largo del periodo de 4 años, se extrajeron 4.898 casos que correspondían a pacientes con Vía Aérea Difícil (VAD) $(0,74 \%$ del total). Determinándose a través de datos demográficos, que es más frecuente esta situación en paciente mayor, sexo masculino y aquellos con un ASA elevada.

Del total de 4.898 casos, la situación "no intubable, no ventilable con mascarilla facial" ocurrió en 455 casos. De ellos se utilizaron DSG de rescate en 86 casos $(18,9 \%)$ y fue exitoso en 54 de ellos (62\% de los casos).

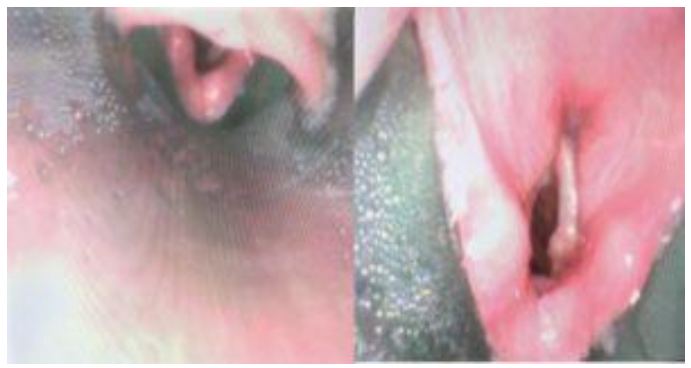

Imagen 2: Visión cuerdas vocales a través de un dispositivo I-Gel

\section{$\underline{\text { Conclusiones }}$}

De los 4.898 casos de VAD se ha utilizado para su manejo un DSG en 607 ocasiones con una tasa de éxito del $65,1 \%$. Además, la colocación de un DSG se intentó en 86 de los 455 casos registrados como paciente "no intubable, no ventilable con mascarilla facial con una tasa de éxito del $62,8 \%$. La tasa de uso de DSG como rescate de un paciente con VAD parece baja, teniendo en cuenta que el uso de estos dispositivos en las guías de VAD 
se introdujo en la década anterior a las fechas recopiladas en la base de datos. Se especula con la posibilidad de que este porcentaje en la fecha actual se haya incrementado.

El éxito descrito en el estudio del $65 \%$ es bastante inferior al descrito por Parmet et al. del 94\% (3). Pero hay que tener en cuenta que este porcentaje representa la práctica clínica diaria recogida en una base de datos, no un estudio enfocado al manejo de los dispositivos supraglóticos.

En conclusión, se ha observado el escaso uso de los DSG dentro de la gestión de una VAD, teniendo en cuenta que están dentro de los algoritmos desarrollados para ello en varias sociedades científicas, entre ellas la Sociedad de Vía Aérea difícil del Reino Unido (DAS).

\section{Comentario}

En la mayor parte de las ocasiones, el manejo de la vía aérea en especialidades como Anestesiología, Cuidados Intensivos o Emergencias, es algo rutinario, y concluye sin complicaciones ni eventos adversos, pero cuando estos ocurren pueden convertirse en una catástrofe, representando la mayor proporción de demandas y litigios relacionados con estas disciplinas médicas (4).

Gracias a las modernas tecnologías que incluye nuevas modalidades de oxigenación (5) (THRIVE y espiración activa), disponibilidad generalizada de capnografía, dispositivos supraglóticos de segunda generación y vídeolaringoscopios, esta incidencia ha disminuido notablemente.

Un reciente estudio describe la "Hegemonía de los ensayos clínicos controlados aleatorizados (ECA), como el Gold Standard de la evidencia médica“, pero en el caso de considerar complicaciones raras(como es el caso de un paciente no intubable, no ventilable) se plantean otras modalidades de investigación (6).

En la gestión de la vía aérea moderna, los dispositivos supraglóticos se utilizan con bastante frecuencia y para una gran variedad de situaciones y grupos de pacientes como pueden ser obesos; aquellos con un grado elevado de Mallampati u otros predictores de riesgo para vía aérea difícil, cirugía abdominal, cirugía en litotomía, rescate de la vía aérea, durante resucitación cardiopulmonar o como canal para una intubación traqueal (7).

Pero desafortunadamente se ha puesto de manifiesto que los ensayos clínicos controlados aleatorizados realizados en pacientes de bajo riesgo son incapaces de responder preguntas sobre seguridad en poblaciones o entornos de alto riesgo. Es aquí donde toman su papel determinante las bases de datos que recogen grandes cantidades de información sobre casos de rutina, y de donde podemos concluir datos de la práctica cotidiana.

Pero estas también presentan su limitación, las más comunes incluyen una población definida (por ejemplo, de un solo hospital o población atípica con características propias) o datos recolectados no bien determinados (si estos son para fines de facturación o administrativos o para fines sanitarios).

Para valorar las complicaciones derivadas del manejo de la vía aérea, las mejor establecidas son:

1.- Base de datos de Anestesia Danesa que recoge medio millón de pacientes de aproximadamente 30 hospitales.

2.- Base de datos Norteamericana de un pequeño número de hospitales 
académicos, que ahora forma el grupo multicéntrico de análisis de resultados perioperatorios.

\section{3.- El ASA Closed Claimed Project, que} ha proporcionado una visión considerable de las complicaciones más graves de manejo de la vía aérea en las últimas dos décadas.

Todas ellas con varios estudios realizados, analizando las complicaciones derivadas del quehacer médico diario.

El estudio que analizamos se ha extraído precisamente de esta primera, y aunque es cierto que presenta una serie de limitaciones que hemos de valorar como son, la no diferenciación de la generación de dispositivo supraglótico que se usa, el uso o no de relajante muscular (para realizar intento óptimo de intubación endotraqueal) y la diferencia de experiencia del diferente personal de manejo de la vía aérea (adjuntos, residentes y enfermería de anestesia), pone de manifiesto $y$ concluye que:

"Los dispositivos supraglóticos no son ampliamente usados en el manejo de la Vía Área Difícil, a pesar de estar fuertemente recomendados en las guías de manejo de estas"

\section{Bibliografía}

1. Apfelbaum JL, Hagberg CA, Caplan RA, Blitt CD, Connis RT, Nickinovich DG, et al. Practice Guidelines for Management of the Difficult Airway. Anesthesiology. 2013;118(2):251-7. (PubMed) (HTML)

2. Frerk C, Mitchell VS, McNarry AF, Mendonca C, Bhagrath R, Patel A, et al.
Difficult Airway Society 2015 guidelines for management of unanticipated difficult intubation in adults. $\mathrm{Br} \mathrm{J}$ Anaesth. 2015; 115(6)827-48 (․ㅏbMed) (MC)

3. Parmet JL, Colonna-Romano P, Horrow JC, Miller F, Gonzales J, Rosenberg H. The laryngeal mask airway reliably provides rescue ventilation in cases of unanticipated difficult tracheal intubation along with difficult mask ventilation. Anesth Analg. 1998;87:661-5. (PubMed)

4. Cook TM, Scott S, Mihai R. Litigation related to airway and respiratory complications of anaesthesia: An analysis of claims against the NHS in England 1995-2007. Anaesthesia. 2010;65:556-63. (ubMed)

5. Patel A, Nouraei SAR. Transnasal Humidified Rapid-Insufflation Ventilatory Exchange (THRIVE): a physiological method of increasing apnoea time in patients with difficult airways. Anaesthesia [Internet]. 2015;70(3):323-9. Available from: http://www.ncbi.nlm.nih.gov/pubmed/253 88828\%0Ahttp://www.pubmedcentral.nih.gov/a $\underline{\text { rticlerender.fcgi?artid=PMC4674986 }}$

6. Moppett IK, Moonesinghe SR, Grocott MP. National audits in the UK: fancy acronyms or real patient benefit? British Journal of Anaesthesia. 2017;118(4) 479-82. (PubMed)

7. Cook TM, Kelly FE. Time to abandon the 'vintage' laryngeal mask airway and adopt second-generation supraglottic airway devices as first choice. Br J Anaesth. 2015; 115(4) 49799 (PubMed)

Correspondencia al autor

Miguel Angel Fernández Vaquero

mferva7@gmail.com

FEA Departamento de Anestesia y Cuidados Intensivos.

Clínica Universidad de Navarra, Madrid.

Aceptado para el blog en junio de 2019 